\title{
Outcomes with Ventricular Assist Device Therapy for Advanced Heart Failure in North Macedonia: First Annual Report
}

\author{
Petar Risteski, ${ }^{1}$ Tanja Angusheva, ${ }^{2}$ Rodney Rosalia, ${ }^{2}$ Sonja Grazdani, ${ }^{3}$ Milka Klinceva, ${ }^{2}$ Venko Filipce, ${ }^{4}$ \\ Marjan Shokarovski, ${ }^{3}$ Aron Frederik Popov, ${ }^{5}$ Sasko Jovev, ${ }^{3 *}$ Zan Mitrev ${ }^{2 *}$ \\ ${ }^{1}$ Department of Thoracic and Cardiovascular Surgery, University Hospital Frankfurt, Frankfurt am Main, Germany; ${ }^{2}$ Department \\ of Cardiovascular Surgery, Zan Mitrev Clinic, Skopje, Republic of North Macedonia; ${ }^{3}$ Department of Cardiovascular Surgery, \\ University St. Cyril and Methodius, Skopje, Republic of North Macedonia; ${ }^{4}$ Department of Neurosurgery, University St. Cyril and \\ Methodius, Skopje, Republic of North Macedonia; ${ }^{5}$ Department of Thoracic and Cardiovascular Surgery, University Medical Centre \\ Tübingen, Tübingen, Germany
}

\section{ABSTRACT}

Introduction: Mechanical circulatory support by a continuous-flow ventricular assist device (VAD) improves survival and quality of life in selected patients with advanced heart failure. Developing countries have been struggling to construct a contemporary and effective health care system to manage advanced heart failure. This observation represents the first annual report on clinical outcomes with VAD for patients with advanced heart failure in the Republic of North Macedonia.

Methods: Data from all patients with VAD implantations between November 2018 and December 2019 were collected. The etiology of the heart failure was dilated cardiomyopathy in 4 patients $(57 \%)$, ischemic cardiomyopathy in $2(28 \%)$, and hypertrophic cardiomyopathy in 1 (14\%). The primary outcome was survival; secondary outcomes included adverse events defined according to the Interagency Registry for Mechanically Assisted Circulatory Support.

Results: A total of 7 patients (85\% males, median age 56 years) received a $\mathrm{VAD} ; 5$ of them received left $\mathrm{VAD}$, and the remaining 2 received biventricular VAD. There were no deaths. Observed morbidity during a mean follow-up of 216 days included 3 bleeding events in 1 patient, 2 patients with superficial driveline infection, and 1 minor stroke and a pump thrombosis, which were treated with VAD exchange. Significant improvement in quality of life, as assessed by the Kansas City Cardiomyopathy Questionnaire and the Functional Independence Measure ${ }^{\mathrm{TM}}$ instrument, was seen with all patients.

Conclusions: Our results demonstrate a successful initiation of the VAD program in the Republic of North Macedonia. Proper training of a dedicated HF team supports the reproducibility of this treatment in developing countries.

Received April 18, 2020; accepted April 27, 2020.

*S. Fovev and Z. Mitrev contributed equally to this work.

Correspondence: Zan Mitrev, MD, FETCS, Department of Cardiovascular Surgery, Zan Mitrev Clinic, St. Bledski Dogovor Nr. 8, Skopje, Republic of North Macedonia; +3892309 1500; fax: +38923104931 (e-mail: zan@zmc.mk).

\section{INTRODUCTION}

Orthotropic heart transplantation has been considered to be the gold standard for treatment of patients with advanced heart failure (HF) in developed countries, with a reported 1 -year survival rate of $86 \%$ [Lund 2015]. Over the last 2 decades, organ donor shortage has promoted mechanical circulatory support (MCS) therapies with ventricular assist devices (VADs) for advanced HF worldwide.

A VAD program was initiated in 2 tertiary cardiac care units in the Republic of North Macedonia in late 2018. The program represents a significant leap forward in the treatment of patients with advanced HF in our developing country, which has historically struggled to keep pace with the rapidly evolving health care landscape, in particular, innovations in cardiac surgery.

This study is the first annual report on the clinical outcomes following the initiation of a VAD program for advanced $\mathrm{HF}$ in the Republic of North Macedonia.

\section{METHODS}

This observational study encompasses 7 consecutive patients who underwent VAD implantations in Skopje, the Republic of North Macedonia, between November 2018 and December 2019. All implantations of permanent MCS devices, including left VAD (LVAD) and biventricular VAD (BiVAD), performed at the Department of Cardiovascular Surgery, University St. Cyril and Methodius, and the Department of Cardiovascular Surgery, Zan Mitrev Clinic (both in Skopje) are included in this observation. Before November 2018, there was no dedicated program on a national level for patients with advanced $\mathrm{HF}$, including heart transplantation, and patients have received optimal medical or conventional surgical therapy [Mitrev 2002].

Data were collected retrospectively in a single online database and were cross-checked by staff from both departments. The database includes detailed information on demographics of the patients, preoperative risk factors, operative details, postoperative hospital course, and early and midterm morbidity and mortality. The local Ethics Committee of Zan 
Mitrev Clinic approved this observational study based on clinical data from both institutions, and the clinical practice complied with the Declaration of Helsinki. All patients submitted signed informed consent.

All implantations were performed for advanced HF refractory to maximal medical therapy, after careful assessment of all investigations and approval from a dedicated HF heart team. We defined a refractory medical condition as persistent or worsening symptoms: namely, an increase in Interagency Registry for Mechanically Assisted Circulatory Support (INTERMACS) status, despite optimal medical therapy, other cardiac interventions, or surgery.

The primary outcome was survival. Secondary outcomes were adverse events defined according to the Interagency Registry for Mechanically Assisted Circulatory Support (INTERMACS) and included major adverse cardiovascular and cerebrovascular events, surgical site and driveline infections, bleeding complications, bacteremia, and pump thrombosis and exchange.

\section{Operative Technique}

After median sternotomy and pericardiotomy, the patient was put on a cardiopulmonary bypass with central arterial and venous cannulation. A needle vent was placed in the ascending aorta and used for deairing. The inflow cannula of the LVAD was implanted after securing the ring on the left ventricular apex, taking care to direct the inflow cannula toward the mitral valve, which was confirmed with transesophageal echocardiography. The outflow graft was anastomosed on the ascending aorta in all patients. A mechanical aortic valve prosthesis was additionally replaced with a biological valve prosthesis in a single patient who underwent a previous aortic valve replacement. In 2 patients with biventricular HF, right VAD (RVAD) was implanted in addition to LVAD. The BiVAD implantation technique used has been previously published [Lavee 2018].

\section{Anticoagulation Regimen}

Intravenous heparin was started in the morning hours of the first postoperative day to maintain an activated partial thromboplastin time of 60 to 75 seconds. Acetylsalicylic acid, $100 \mathrm{mg}$ p.o., was administered as soon as the patient was extubated. Therapy with oral vitamin $\mathrm{K}$ antagonist was started after removal of the chest drainage tubes and continued indefinitely to maintain an international normalized ratio (INR) of 2.5 to 3.0. Heparin was discontinued as soon as INR reached the target range.

\section{Follow-Up}

All patients have been systematically followed in our outpatient clinics once per month with clinical evaluation, transthoracic echocardiography assessment, and blood and coagulation tests. No patient was lost to follow-up. Median follow-up was 216 days (range 118 to 352). All patients were required to answer the Kansas City Cardiomyopathy Questionnaire (KCCQ QoL survey) [MacIver 2012] and the Functional Independence Measure ${ }^{\mathrm{TM}}$ (FIM instrument, Guide for the Uniform Data Set for Medical Rehabilitation, 1996) [Fiedler 1996], as recommended.

\section{Statistical Analysis}

Statistical reporting was performed according to the SAMPL guidelines [Lang 2013]. Categorical parameters were summarized as absolute numbers and percentages. Continuous data are shown as mean $\pm \mathrm{SD}$ or median and interquartile range. Continuous variables were evaluated using the D'Agostino and Pearson normality test. Comparisons of preoperative versus postoperative data (KCCQ QoL survey) were performed using a paired $\mathrm{t}$ test or Wilcoxon signed rank test for nonparametric data. Repeated-measures 1-way analysis of variance (ANOVA) was used to evaluate creatinine/glomerular filtration rate, bilirubin, and FIM scores. The data were analyzed with GraphPad Prism, version 7.03.

\section{RESULTS}

Seven patients (median age 56 years, range 29 to 59) were included in the current case series. The etiology of the heart failure was dilated cardiomyopathy in 4 patients (57\%), ischemic cardiomyopathy in 2 (28\%), and hypertrophic cardiomyopathy in 1 (14\%). History of previous cardiac surgery was present in 1 patient (14\%). Table 1 summarizes the preoperative patient demographics and clinical characteristics. Preoperative echocardiography investigations are presented in Figure 1.

\section{Early Results}

The patients were preoperatively admitted to the intensive care unit to optimize organ function. Preoperative temporary mechanical circulatory support was not required in any patient, as all patients were in INTERMACS profiles 3 and 4 .

Five patients received LVAD; HeartWare (HW, Medtronic, Minneapolis, MN) was implanted in 2 patients, and HeartMate 3TM (HM3, Abbott, Chicago, IL) was implanted in 5 patients. Two of these patients simultaneously received

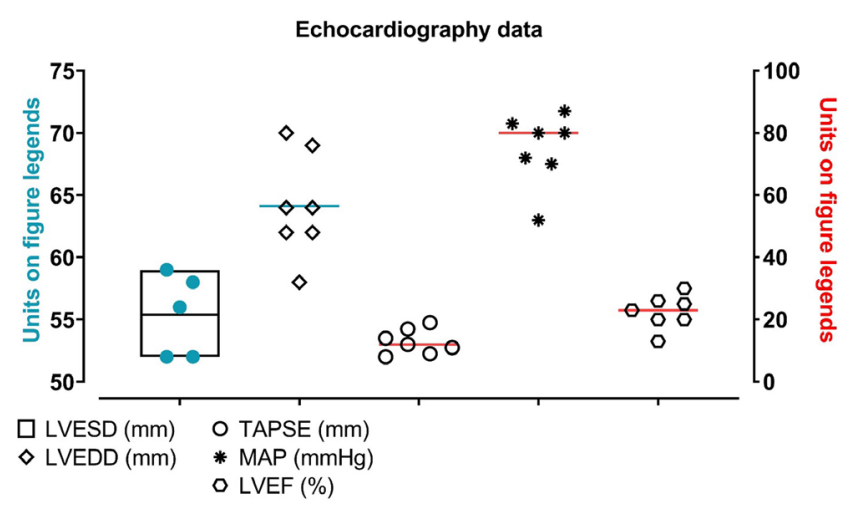

Figure 1 Preoperative Echocardiography assessment of the study group who underwent VAD implantation as destination therapy for chronic heart failure. (LVESD, Left Ventricular End-Systolic Dimension; LVEDD, Left Ventricular end-diastolic Dimension; TAPSE, Tricuspid Annular Plane Systolic Excursion; MAP, mean arterial pressure; LVEF, left ventricular ejection fraction). 
Table 1. Patient Characteristics, Demographics, and Clinical Observations

\begin{tabular}{lc}
\hline Characteristic & Value \\
\hline Age $(y)$ & $56(29,59)$ \\
Female sex & $1(14.3)$ \\
Median height (cm) & 175.0 \\
Median weight (kg) & 80.00 \\
BMI (kg/m²) & $23.78(19.27,28.37)$ \\
Tricuspid insufficiency & $6(85.7)$ \\
Mitral insufficiency & $7(100)$ \\
Diabetes mellitus & $4(57.1)$ \\
Arterial hypertension & $5(71.4)$ \\
Peripheral vascular disease & $1(14.3)$ \\
Chronic obstructive pulmonary disease & $1(14.3)$ \\
Chronic renal insufficiency & 0 \\
NYHA III & $3(42.7)$ \\
NYHA IV & $4(57.1)$ \\
INTERMACS III & $5(71.4)$ \\
INTERMACS IV & $2(28.6)$ \\
HeartMate III & $5(71.4)$ \\
HeartWare & $2(28.6)$ \\
ICD & $2(28.6)$ \\
Previous cardiac surgery & $1(14.3)$ \\
\hline & \\
\hline
\end{tabular}

*Data are median (minimum, maximum) or $\mathrm{n}(\%)$ unless noted otherwise. BMI indicates body mass index; ICD, implanted cardioverter defibrillator; NYHA, New York Heart Association functional class.

additional HM3 as a permanent RVAD. Additional procedures included aortic valve prosthesis replacement in 1 patient, and partial thyroidectomy. Median cardiopulmonary bypass time was 94 minutes (range 67 to 228). Median ventilation time was 35.5 hours (range 2 to 68 ), and intensive care unit stay was $18.4 \pm 3.6$ days. We observed postoperative courses devoid of significant complications or mortality (Table 2); 2 patients underwent reopening for bleeding.

Neurologic morbidity was not observed in any patient during the initial hospital stay. A single patient had 3 episodes of small bowel bleeding during the initial admission. The first and second bleeding events were unsuccessfully treated with coiling of the end-stream branch of the mesenteric artery. After the third bleeding event, the patient underwent laparotomy and bowel resection to completely resolve this complication. The histology report showed angiodysplastic lesion of the small bowel mucosa. Renal (Figure 2A) and liver (Figure 2B) failure were not observed. Morbidity during the initial hospital stay included 1 bacteremia and 1 early driveline infection, which was successfully treated with topical and systemic antibiotics. No
Table 2. Early Postoperative Results $(\mathrm{N}=7)^{*}$

\begin{tabular}{lc}
\hline Item & Value \\
\hline Readmission within 1 month after discharge & $1(14.3)$ \\
Major infections & $1(14.3)$ \\
Superficial surgical site infection & 0 \\
Deep surgical site infection & 0 \\
Bacteremia & 1 \\
Driveline infections & $1(14.3)$ \\
Neurological complication & 0 \\
Gastrointestinal bleeding & $1(14.3)$ \\
Multiorgan failure & 0 \\
Acute renal failure & 0 \\
Hepatic failure & 0 \\
Malignant cardiac arrhythmia & 0 \\
Pump thrombosis & 0 \\
Thromboembolism & 0 \\
\hline
\end{tabular}

*Data are $\mathrm{n}(\%)$. Operative mortality is defined in all 3 Society of Thoracic Surgeons databases as (1) all deaths, regardless of cause, occurring during the hospitalization in which the operation was performed, even if after 30 days (including patients transferred to other acute care facilities); and (2) all deaths, regardless of cause, occurring after discharge from the hospital, but before the end of the 30th postoperative day.

further complications were reported. Hospital stay was $26.6 \pm 7.8$ days.

\section{Midterm Results}

All patients entered follow-up; the median follow-up at the time of the current analysis was 7.80 months (range 4.23 to 8.42 ) based on an accumulated total of 32 patient-months. We did not observe mortality during follow-up. Two patients were readmitted with driveline infections and were successfully treated with combined topical and oral antibiotics. Surgical intervention on the driveline was not required (Table 3).

Another patient was readmitted with a nondisabling minor ischemic stroke and pump thrombosis, which we resolved with systemic administration of fibrinolytic agents; we readmitted the same patient on 155 th postoperative day with a repeat pump and outflow graft thrombosis. The underlying cause of the multiple events is noncompliance with the recommended medical therapies as prescribed; the patient did not take his oral anticoagulants for 1 week. He underwent pump exchange through an anterolateral minithoracotomy and partial upper mini-sternotomy. A superficial thoracotomy wound dehiscence was the only complication after this event; after the intervention and a reprimand by the treating physician, he experienced an uncomplicated postoperative recovery.

We observed substantial improvement in the quality of life of the cohort; based on the KCCQ-12 survey, all patients noted markedly better scores for physical function, frequency 
A
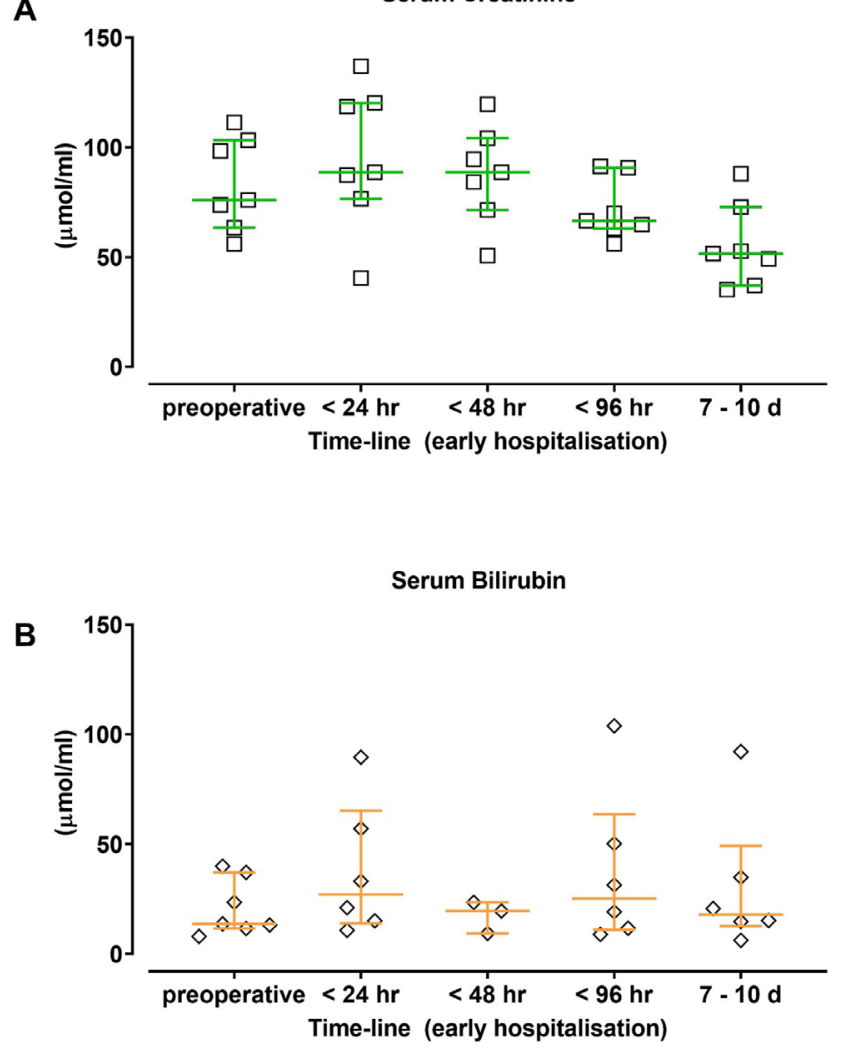

Figure 2 Perioperative kidney (A) and liver function (B) including sequential analysis of systemic creatinine and bilirubin levels in the study population. ANOVA Creatinine, treatment effect, $p=0.0094$. ANOVA Bilirubin, treatment effect, $\mathrm{P}>0.05$

and severity of symptoms, social function, and self-efficacy (P $<.0001$ ) (Figure 3A).

Along the same lines, our patients' functional independence scores gradually improved over time after hospitalization (Figure 3B). The patients reported increased day-to-day independence at discharge, with a mean difference of 10.0 (95\% confidence interval 0.26 to 19.74$], \mathrm{P}=.045$, and at follow-up, 20.7 ( 0.86 to 40.57 ), $\mathrm{P}=.043$, since surgery.

\section{DISCUSSION}

The study aimed to investigate the preliminary results of VAD implantations in the Republic of North Macedonia from 2 cardiac centers. This country, like many developing countries, has struggled to establish a contemporary, comprehensive, and effective health care system to manage the increasing burden of heart failure, mainly because of nonmedical factors such as poor socioeconomic status as well as regional and national political instability. Unhealthy lifestyle is another factor, and according to the Systematic Coronary Risk Evaluation (SCORE), North Macedonia belongs to the very-high-risk group of countries for cardiovascular diseases
Table 3. Midterm Postoperative Course, Clinical Events, and Major Complications Requiring Readmission Within 30 to 90 Days*

\begin{tabular}{lc}
\hline Item & Value \\
\hline Reoperation (LVAD exchange) & $1(14.3)$ \\
Bleeding events & $2(28.6)$ \\
Major infections & $1(14.3)$ \\
Bacteremia & 0 \\
Driveline infections & 0 \\
Pump-related infections & 1 \\
Neurological complication & $1(14.3)$ \\
Gastrointestinal bleeding & $1(14.3)$ \\
Multiorgan failure & 0 \\
Acute renal failure & 0 \\
Acute hepatic failure & 0 \\
Thrombosis & $1(14.3)$ \\
Thromboembolism & $1(14.3)$ \\
Pump exchange & $1(14.3)$ \\
\hline
\end{tabular}

*Data are $n(\%)$.

[Cooney 2016]. However, cardiovascular disease burden is a global phenomenon, the prevalence of HF is increasing substantially in developed countries as well, and despite improvements in medical therapy, HF still carries a poor prognosis [Ponikowski 2016].

Mechanical circulatory support by a continuous-flow left ventricular assist device improves survival and quality of life in selected patients [Popov 2012]. The experience regarding long-term outcomes in these patients is growing, as the waiting time for heart transplantation is increasing owing to the shortage of donor hearts; therefore left ventricular assist device placement is a reasonable therapeutic option for patients with end-stage heart failure [Popov 2012]. This therapeutic option is especially important in countries without an active heart transplantation program established by an official governmental body or other comparable institution, which results in almost in all cases toward a destination therapy.

With this work, we highlight our initial experience with a national VAD program. The report is unique for our country; moreover, to the best of our knowledge, it is only the second study in terms of contemporary heart failure treatment with long-term mechanical assist devices in the Balkans, the first being published recently [Nestorovic 2018]. After systematic and rigorous training of all dedicated staff, on-site and abroad, the program started in November 2018.

Our study demonstrates that even with low numbers of LVAD implantations in a newly established heart failure program, the outcome can be comparable with the recent analysis from the United States INTERMACS registry of LVAD, 
A

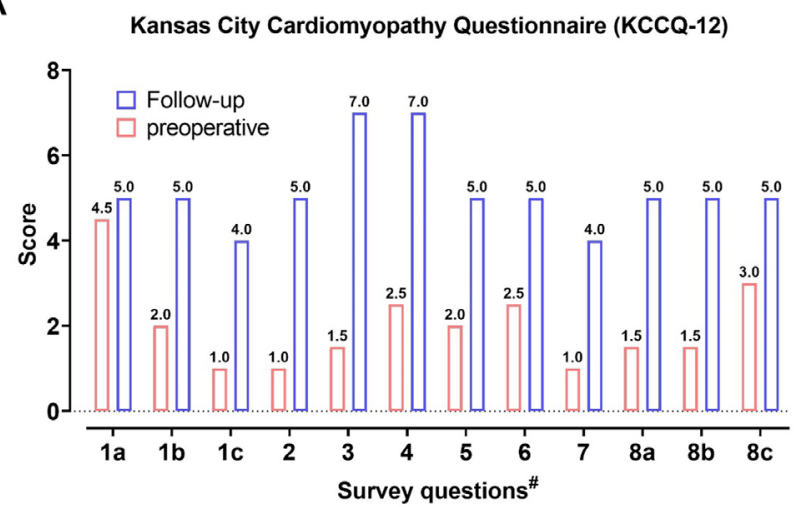

B

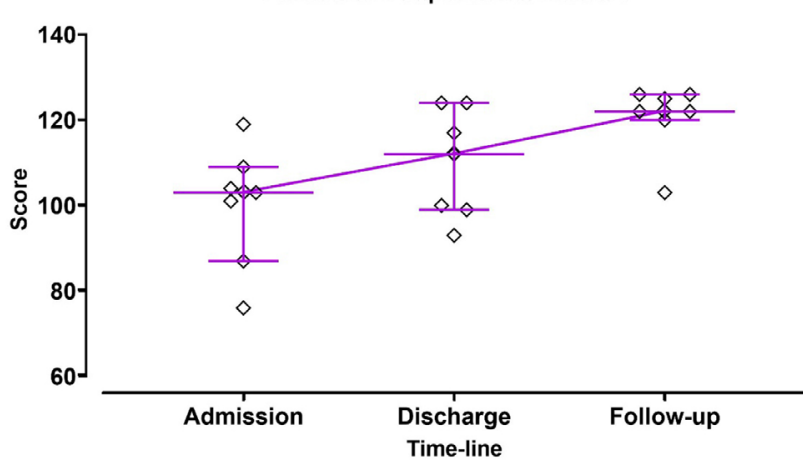

Figure 3 Pre- and postoperative quality-of-life assessment; quality of life was measured using two independent methods A Kansas City Cardiomyopathy Questionnaire (KCCQ-12) and B The Functional Independence Measure (FIM)TM (Guide for the Uniform Data Set for Medical Rehabilitation, 1996). KCCQ-12, Mixed-effects model, treatment effect $p<0.0001$. FIMTM, treatment effect, repeated measures ANOVA, $p$ $=0.0275$

which shows a 1-year survival of $80 \%$ and 2-year survival of 70\% [Goldstein 2019; Kormos 2019].

Several factors might explain our VAD-program's promising start: in the 12 months preceding the first surgery, all members of the HF team at both centers underwent training abroad (Berlin, Germany) in terms of LVAD treatmen (clinical investigations, surgical practice, mechanical circulatory support, intensive care treatment, postoperative care, and patient selection). We received on-site and remote support by experts from the cardiac surgery center-of-excellence clinics from Bad Neustadt an der Saale, Frankfurt, and Tübingen (Germany) and Ljubljana, Slovenia.

Adequate patient selection is one of the most critical factors to gain optimal results; therefore, our findings are consistent with previous studies [Michaels 2019]. Apart from that, the US INTERMACS registry of LVAD demonstrates that the so-called crash and burn patients (INTERMACS profile 1) have the worst outcome [Goldstein 2019]. We have so far operated only on INTERMACS profile 3 and profile 4 patients, which underlines the observed survival in this series.
Correspondingly, we have not seen typical complications such as disabling stroke, kidney failure, or postoperative right heart failure in patients with LVAD, or major bleeding, which accompanies an inferior outcome in LVAD patients [Nestorovic 2018; Acharya 2017; Yalcin 2020; Birks 2017]. However, we observed 1 patient with gastrointestinal bleeding in our follow-up. Gastrointestinal bleeding is a considerable problem after LVAD implantation and has an incidence estimated between $15 \%$ and 61\% [Gurvits 2017]. LVAD-associated gastrointestinal bleeding is associated with significant morbidity, costs, and readmissions [Guha 2015]. It has been hypothesized that antithrombotic therapy, angiodysplasia, and the continuous-flow pump itself lead to a significant risk to develop gastrointestinal bleeding [Birks 2017]. Our patient received successful interdisciplinary treatment to deal with the gastrointestinal bleeding and in the ongoing followup. He is free from recurrent bleeding.

Another typical complication that we experienced was LVAD thrombosis (1 patient). This complication is rare $(1.4 \%$ to $11 \%$ within 2 years) [Pagani 2009]. The pathophysiology behind this complication is not fully understood; however, it has been hypothesized that internal high shear stress, device material and surface characteristics, chronic infection, and malposition of the device or inadequate anticoagulation are responsible [Goldstein 2013]. In our case, the patient was not correctly anticoagulated and experienced rapid hemodynamic deterioration. LVAD thrombosis was confirmed, and the patient underwent an LVAD pump exchange via left-sided thoracotomy and partial upper ministernotomy without any complications. This surgical approach has been described for complex LVAD exchanges [Radwan 2018]. However, there is no clear recommendation favoring thrombolysis or surgery in cases of LVAD thrombosis. A systematic review article with 43 individual trials thematically considering medical therapy versus early LVAD exchange in the circumstance of device thrombosis was recently published [Luc 2019]. This meta-analysis showed a benefit for device exchange in terms of success rate compared with medical therapy, resulting in significantly lower 30-day mortality and a significantly higher rate of freedom from rethrombosis compared with thrombolysis. This finding is in line with our experience, although only encountered once, we avoided severe hemorrhage, hemorrhagic stroke, intracranial bleed, and unsuccessful thrombolysis [Luc 2018].

There are several limitations to be considered when interpreting the present study. First, these data are preliminary, and the observation time is not comparable to that of international studies. Second, the number of LVAD implantations is quite low and included only patients with INTERMACS profiles 3 and 4 . Even in this patient population, we have been able to deal with difficult situations such as right heart failure with a permanent RVAD implantation, preoperative LVAD implantation including aortic valve prosthesis replacement, and pump thrombosis with complex redo surgery and eventually pump exchange. Despite all of this, we were able to reach $100 \%$ survival in this challenging setting. The strength of the study is that this is the first multicenter study reporting on outcome in LVAD patients in whom the device was implanted as a bridge to destination. Patient follow-up was complete in our centers, minimizing the risk of missing data. 
In conclusion, in our experience, based on 5 LVAD and 2 BiVAD implantations, the use of VAD for advanced HF demonstrated survival after a mean follow-up of 296 days. These results support the use of VAD as a destination therapy or bridge to transplantation in selected patients. Proper training of a dedicated HF team proves the reproducibility of this treatment in developing countries.

\section{ACKNOWLEDGMENTS}

The authors thank the Ministry of Health of the Republic of North Macedonia for initiating, supporting, and establishing the VAD program. We are in debt to Professor Dr I. Knežević for his unlimited support and hands-on assistance during the early phases of the VAD program.

\section{REFERENCES}

Acharya D, Loyaga-Rendon R, Morgan CJ, et al. 2017INTERMACS analysis of stroke during support with continuous-flow left ventricular assist devices: Risk factors and outcomes. JACC Heart Fail 5:703-711.

Birks EJ. 2017. Stopping LVAD bleeding: A piece of the puzzle. Circ Res 121:902-904.

Cooney MT, Selmer A, Lindman A, et al. 2016. Cardiovascular risk estimation in older persons: SCORE O.P. Eur J Prev Cardiolog 23:1093-1103.

Fiedler RC, Granger CV. The functional independence measure: A measurement of disability and medical rehabilitation. In: Chino N, Melvin JL, eds. Functional Evaluation of Stroke Patients. Tokyo: Springer; 1996:75-92.

Goldstein DJ, John R, Salerno C, et al. 2013. Algorithm for the diagnosis and management of suspected pump thrombosis. J Heart Lung Transplant 32:667-670.

Goldstein DJ, Meyns B, Xie R, et al. 2019. Third annual report from the ISHLT mechanically assisted circulatory support registry: A comparison of centrifugal and axial continuous-flow left ventricular assist devices. J Heart Lung Transplant 38;352-362.

Guha A, Eshelbrenner CL, Richards DM, Monsour HP Jr. 2015. Gastrointestinal bleeding after continuous-flow left ventricular device implantation: Review of pathophysiology and management. Methodist Debakey Cardiovasc J 11:18-23.

Gurvits GE, Fradkov E. 2017. Bleeding with the artificial heart: Gastrointestinal hemorrhage in CF-LVAD patients. World J Gastroenterol 23:3945-3953.

Kormos RL, Cowger J, Pagani FD, et al. 2019. The Society of Thoracic Surgeons Intermacs database annual report: Evolving indications, outcomes and scientific partnerships. J Heart Lung Transplant 38:114-126.

Lang T, Altman D. Basic statistical reporting for articles published in clinical medical journals: The SAMPL Guidelines. In: Smart P, Maisonneuve
H, Polderman A, eds. Science Editors' Handbook. Exeter, UK: European Association of Science Editors; 2013.

Lavee J, Mulzer J, Krabatsch T, Marasco S, McGiffin D, Garbade J, Schmitto JD, Zimpfer D, Potapov EV. 2018. An international multicenter experience of biventricular support with HeartMate 3 ventricular assist systems. J Heart Lung Transplant 37:1399-1402.

Luc JGY, Tchantchaleishvili V, Phan K, Dunlay SM, Maltais S, Stulak JM. 2019. Medical therapy as compared to surgical device exchange for left ventricular device thrombosis. A systematic review and meta-analysis. ASAIO J 65:307-317.

Lund LH, Edwards LB, Kucheryavaya AY, Benden C, Dipchand AI, Goldfarb S, et al. 2015. The Registry of the International Society for Heart and Lung Transplantation: Thirty-second official adult heart transplantation report-2015; focus theme: early graft failure. J Heart Lung Transplant 34:1244-54.

Maclver J, Ross HJ. 2012. Quality of life and left ventricular device support. Circulation 126:866-874.

Michaels A, Cowger J. 2019. Patient selection for destination LVAD therapy: Predicting success in the short and long term. Current Heart Fail Rep 16:140-149.

Mitrev Z, Anguseva T, Vasileva A, Hristov N, Risteski P. 2002. Transplantation or alternative surgical treatment of patients with ischemic dilative cardiomyopathy and aneurysmatic dilation of the left ventricular cavity. Int J Artif Organs 25(4):321-326.

Nestorovic E, Schmitto JD, Kushwaha SS, et al. 2018. Successful establishment of a left ventricular assist device program in an emerging country: One year experience. J Thoracic Dis 10(suppl 15):S1743-S1750.

Pagani FD, Miller LW, Russell SD, et al. 2009. Extended mechanical circulatory support with a continuous-flow rotary left ventricular assist device. J Am Coll Cardiol 21:54:312-321.

Ponikowski P, Voors AA, Anker SD, et al. 2016. ESC guidelines for the diagnosis and treatment of acute and chronic heart failure: The Task Force for the Diagnosis and Treatment of Acute and Chronic Heart Failure of the European Society of Cardiology (ESC). Developed with the special contribution of the heart Failure Association (HFA) of the ESC. Eur Heart J 37:2129-2200.

Popov AF, Hosseini MT, Zych B, et al. 2012. Clinical experience with HeartWare left ventricular assist device in patients with end-stage heart failure. Ann Thoracic Surg 93:810-815.

Radwan M, Risteski P, Hoffmann R, Popov AF. 2018. Repeat left ventricular device exchange with inflow or outflow correction for recurrent pump thrombosis and cerebral haemorrhage through limited incisions. Eur J Cardiothorac Surg 54:781-783.

Yalcin YC, Muslem R, Veen KM, et al. 2020. Impact of continuous flow left ventricular assist device therapy on chronic kidney disease: A longitudinal multicenter study. J Card Fail 26:333-341. 